\title{
Late-Appearing Abscesses after Injections of Nonabsorbable Hydrogel Polymer for HIV-Associated Facial Lipoatrophy
}

\author{
Derek H. Jones, MD, ${ }^{*}$ Alastair Carruthers, MD ${ }^{\dagger}$ Rebecca Fitzgerald, MD ${ }^{\ddagger}$ \\ Peter G. Sarantopoulos, MD ${ }^{\S}$ and Scott Binder, $\mathrm{MD}^{\S}$
}

The authors have indicated no significant interest with commercial supporters.

\begin{abstract}
A variety of injectable fillers have been employed to correct the stigmatizing facial lipoatrophy associated with HIV infection. ${ }^{1,2}$ A novel filler is Bio-Alcamid (Bio-Alcamid, Polymekon, Milan, Italy), which has been described as an injectable "endoprosthesis." 3 This nonbiodegradable filler is composed of $96 \%$ water and $4 \%$ synthetic polymer polyalkylamide and is purported to be biologically inert. ${ }^{3,4}$ BioAlcamid is intended for large-volume augmentation as may be necessary in hemifacial lipoatrophy (Romberg's disease) or HIV-associated lipoatrophy. ${ }^{3}$ Bio-Alcamid is intended for injection into the subcutaneous space in a bolus form. Fibroplasia ensues around the periphery of the balloon-like implant creating an injectable "prosthesis." Recent reports have documented the success of Bio-Alcamid for the treatment of HIV facial lipoatrophy. ${ }^{5,6}$ We report a series of five patients who have received Bio-Alcamid for HIV associated lipoatrophy and who have developed late-appearing streptococcal bacterial abscesses. In all cases the abscesses resolved with incision and drainage and antibiotic therapy.
\end{abstract}

\section{Case Reports}

\section{Case 1}

A 46-year-old HIV-positive male with a history of facial lipoatrophy presented with 3 days of acute swelling, redness, and tenderness of the left cheek.
He had received a series of facial Bio-Alcamid injections 3 years prior at a Tijuana, Mexico, clinic known to specialize in Bio-Alcamid injections for treatment of HIV facial lipoatrophy. The patient reported no problems with the implant until he underwent a dental procedure where the dentist suspected that the Bio-Alcamid implant may have been punctured with a needle used to deliver intraoral anesthesia. Within 24 hours after the dental procedure, he developed acute tenderness and swelling of the left cheek at which point $875 \mathrm{mg}$ of amoxicillin clavulanate three times daily was initiated. He was referred to our clinic (D.H.J.) 3 days later when the apparent abscess failed to respond to antibiotic therapy.

Physical exam revealed a healthy male in no acute distress. There was an $8.5 \times 7-\mathrm{cm}$ tender, fluctuant abscess on the left cheek. The HIV infection was well controlled on antiretroviral therapy, with a recent CD 4 count of 500 and a viral load of 20,000. An incision and drainage procedure was performed with a No. 11 blade stab incision under 1\% lidocaine with epinephrine. Copious amounts of thick, purulent, yellow contents were drained. Routine culture of the drained material was negative, while an anaerobic culture grew $2+$ Propionibacterium acnes (note that the patient was taking amoxicillin clavulanate at the time of the incision and drainage procedure). Routine histology of the drained

*Division of Dermatology and ${ }^{\S}$ Department of Pathology, David Geffen School of Medicine, University of California at Los Angeles, Los Angeles, California; ${ }^{\dagger}$ Department of Dermatology, University of British Columbia, Vancouver, British Columbia, Canada; ${ }^{\ddagger}$ Private Practice, Los Angeles, California 
material revealed synthetic material surrounded by sheets of neutrophils, consistent with contents of an abscess. The Gram stain revealed Gram-positive cocci in small clusters and small chains, with Streptococcus sp. being the presumed organism. Within 1 week, the abscess had completely resolved with evident return of the facial lipoatrophy on the left side (Figure 1).

\section{Case 2}

In 2002, at the age of 58, an HIV-positive male reported receiving a series of facial Bio-Alcamid injections at the same Tijuana, Mexico, clinic as in Cases 1 and 4. In December 2005, the patient underwent an incision and drainage procedure at our clinic (R.F.) with a No. 11 blade under $1 \%$ lidocaine without epinephrine to alleviate persistent subcutaneous lumpiness and uneven correction that had persisted since the injections were completed. The patient tolerated the procedure well, but approximately 6 weeks later developed acute swelling, tenderness and redness of the right cheek. Another incision and drainage procedure was performed, and copious amounts of purulent, thick, yellow contents were drained. The patient was started on $500 \mathrm{mg}$ of cephalexin orally four times daily. Within 24 hours after the procedure, however, erysipelas-like changes and severe swelling were noted on the right cheek (Figure 2A). The patient required hospitalization for IV vancomycin and multiple incision and drainage procedures. He was discharged home 4 days later on oral clindamycin. A routine culture of the drained contents grew heavy $\alpha$-Streptococcus, viridans group. Within 10 days, the abscess showed complete resolution (Figure 2B). The patient reported that the right side "returned to a quite normal look." In April 2006, however, the right cheek, per the patient's report, acutely "puffed out again" and failed to respond to oral antibiotics. The swelling persisted. The patient denied any fever, tenderness, or redness of the right cheek, but continued to tolerate the persistent, stable swelling until September 2006 when he presented to our clinic (D.H.J.). He was placed on $500 \mathrm{mg}$ of cephalexin orally four times daily for 1 week. An incision and drainage procedure was then performed with a stab incision under $1 \%$ lidocaine with epinephrine. A
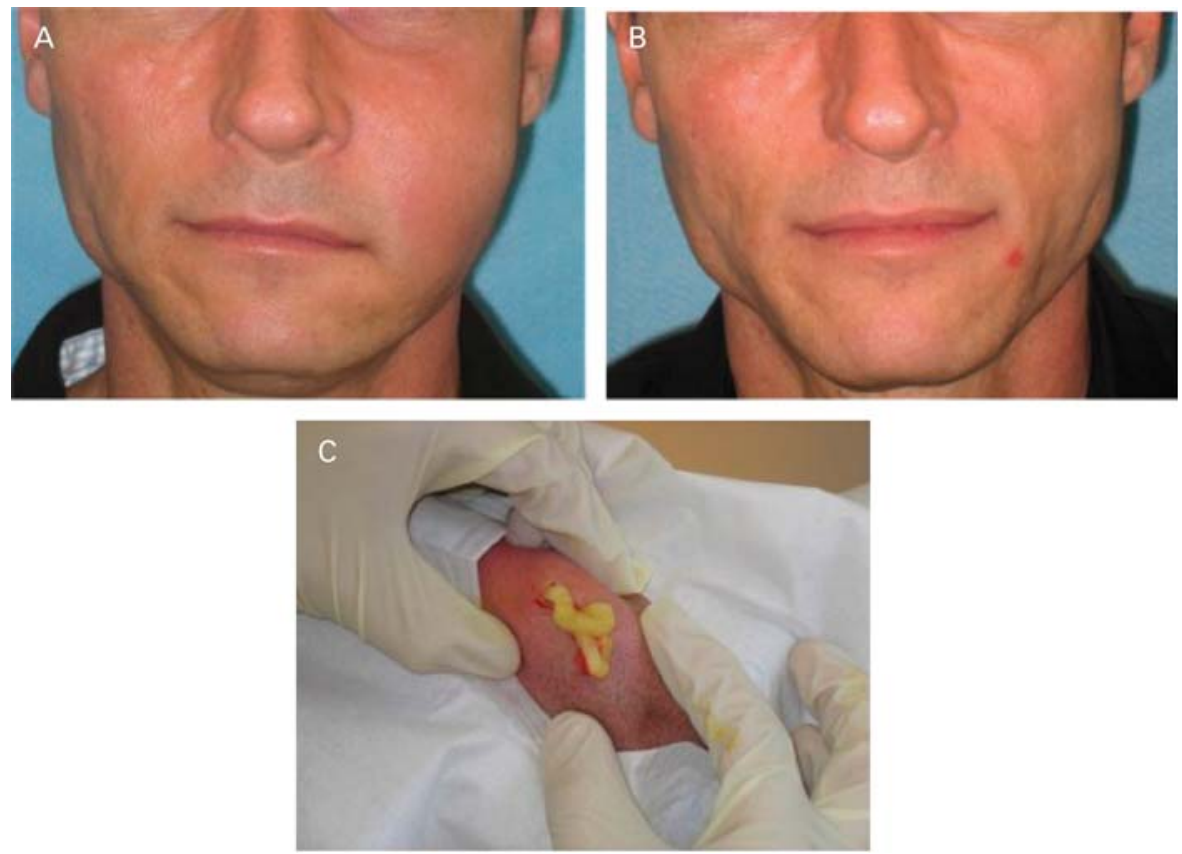

Figure 1. Case 1: (A) acute abscess; (B) 1 month after incision and drainage; (C) drainage of purulent infected implant material. 

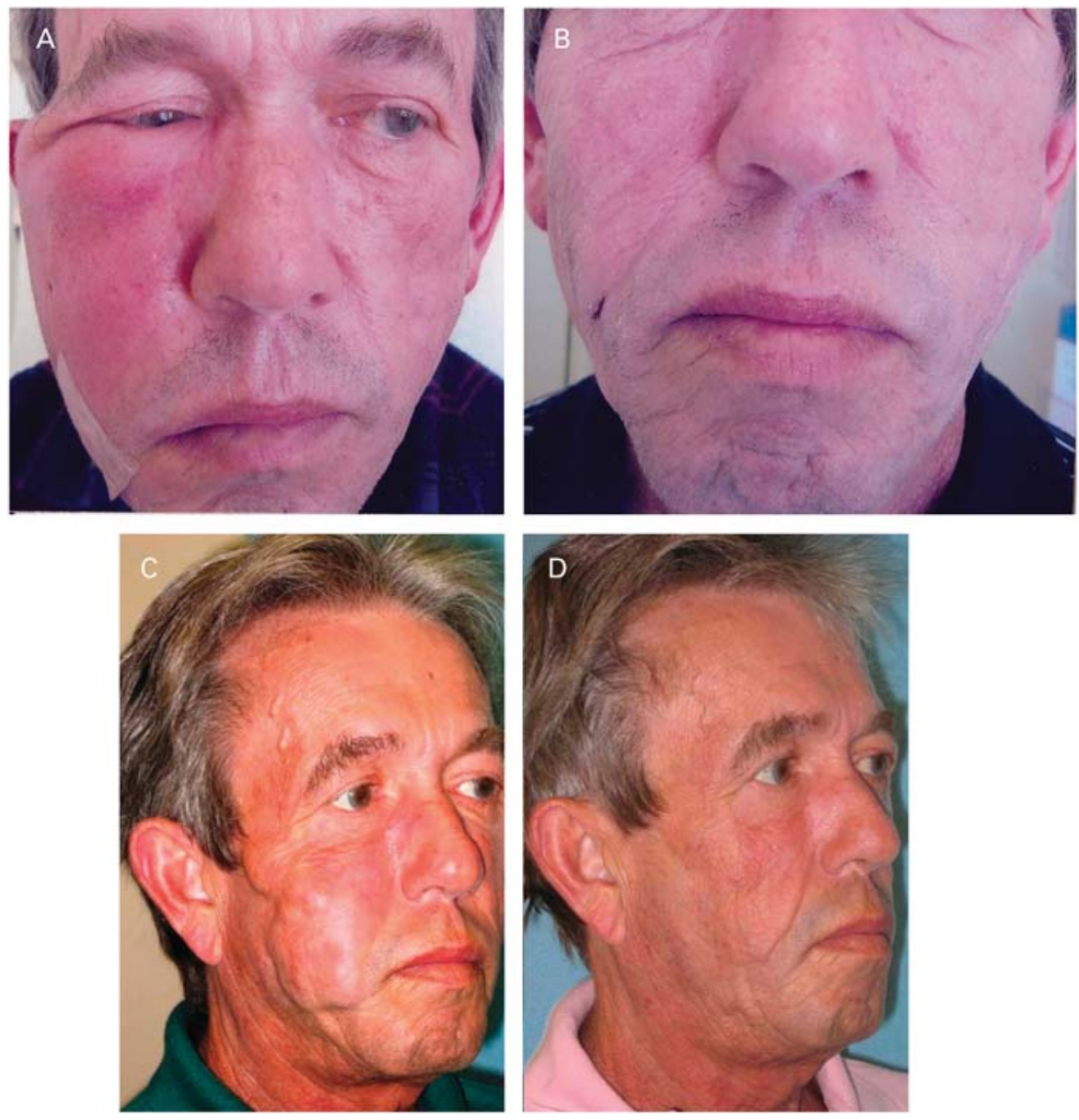

Figure 2. Case 2: (A) presentation of acute abscess in February 2006; (B) 10 days after surgical incision and drainage and antibiotic therapy; (C) presentation of recurrent, chronic abscess of 5 months duration; (D) appearance 1 week post incision and drainage.

copious amount $(>100 \mathrm{~mL})$ of yellow, thin, purulent fluid was drained. A pressure dressing was applied for 48 hours to prevent seroma formation. Culture and sensitivity studies performed on the drained material were negative. Histologic analysis revealed synthetic implant material in association with chronic inflammation including foreign body giant cells, scattered neutrophils, macrophages, and red blood cells. Gram stain revealed rare coccobacillary forms, and GMS and AFB stains were negative for fungal and acid fast organisms. The patient noted complete resolution of the problem on follow-up at 1 week.

\section{Case 3}

An HIV-positive male with facial lipoatrophy underwent Bio-Alcamid injections in our clinic (A.C.).
He was given $1000 \mathrm{mg}$ of cephalexin preoperatively and a total of $25 \mathrm{~mL}$ of Bio-Alcamid was injected into the cheeks and temples. He was given $500 \mathrm{mg}$ of cephalexin orally three times daily for 5 days postoperatively. The patient noted injection site swelling and mild tenderness that slowly resolved over a 2 -week period. Approximately 5 weeks after the first injection, the patient underwent a touch-up injection with Bio-Alcamid $(2.5 \mathrm{~mL}$ to the right cheek and $1.5 \mathrm{~mL}$ to the left cheek) to correct slight contour irregularity. At the 4-month follow-up, the patient was very pleased with the correction and no adverse events were noted. At approximately 5 months after the first injection, the patient experienced an acute episode of severe swelling, warmth, and tenderness of the left cheek. A total of $100 \mathrm{mg}$ of 
minocycline orally twice daily was prescribed by a physician at a walk-in clinic. Three days later, the swelling persisted and the antibiotic was changed to $300 \mathrm{mg}$ of clindamycin orally three times daily. Eight days after the acute swelling developed, an incision and drainage procedure was undertaken to alleviate the persistent abscess. Twenty-five milliliters of yellow purulent material was aspirated from the left cheek. Culture and sensitivity studies of the drained aspirated material grew heavy Group G Streptococcus. The abscess promptly resolved after the drainage procedure and no further adverse events have been noted with up to 1 year of follow-up (Figure 3).

\section{Case 4}

A 42-year-old HIV-positive male with a CD4 count of 500 and an undetectable viral load reported receiving a series of Bio-Alcamid injections to treat facial lipoatrophy at the same Mexican clinic as in Cases 1 and 2. Approximately 2 years after the injections, the patient reported undergoing at the same clinic an incision and drainage procedure on the right cheek only to correct lumpiness and contour irregularity. Approximately 6 months after the incision and drainage procedure, the patient reported that he acutely (overnight) developed massive swelling of the right cheek with associated erythema and extreme tenderness. He denied fever or drainage. At the time the patient was traveling outside the United States, and he was treated with an unknown oral antibiotic and nonsteroidal antiinflammatory medication. He reported that the tenderness and redness resolved over a 2-week period. The swelling persisted, however, without other symptoms of infection (tenderness, fever, drainage, redness) for an additional 9 months until he presented to our office (D.H.J.) for treatment. On
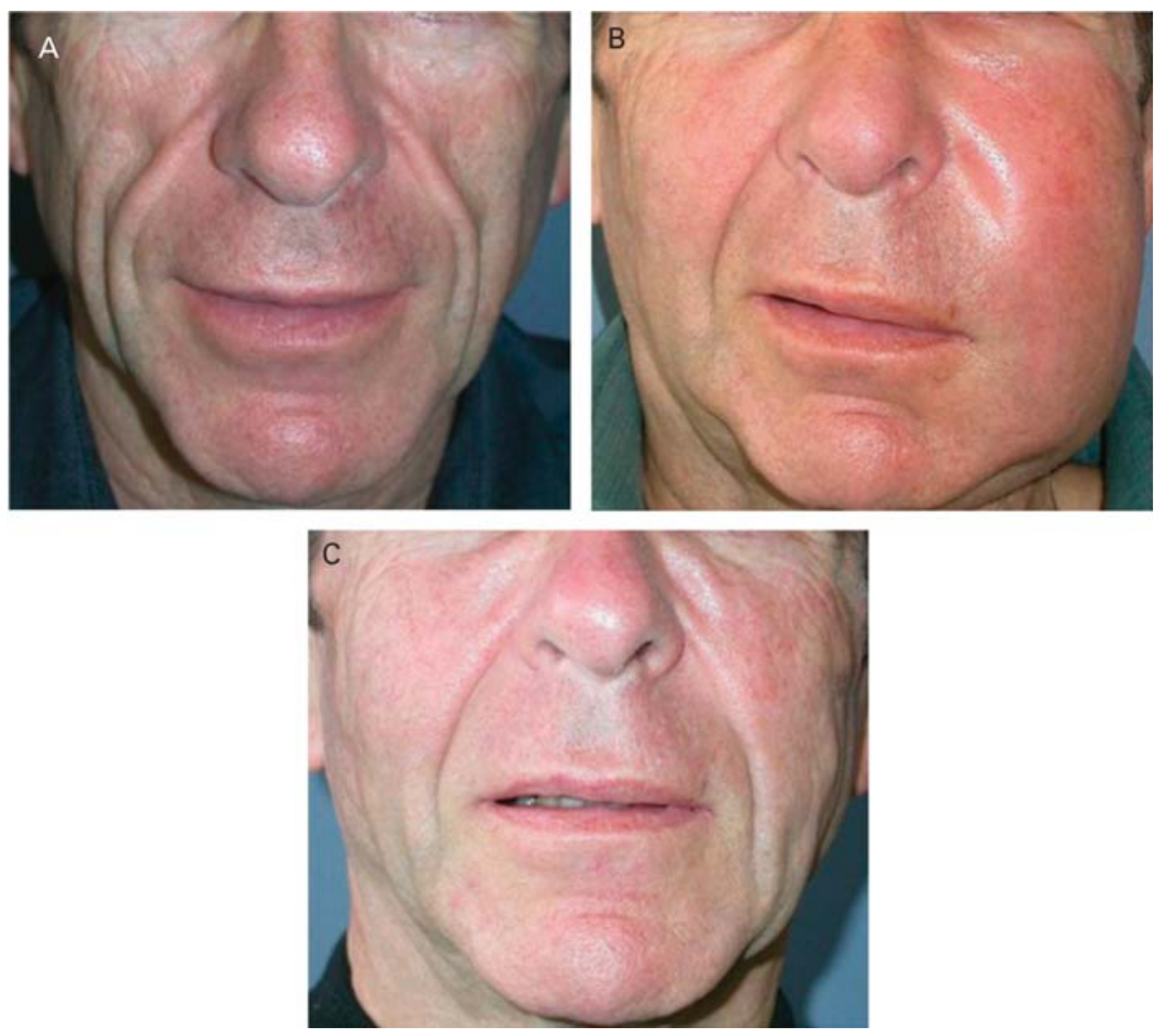

Figure 3. Case 3: (A) baseline before Bio-Alcamid (January 2005); (B) presentation of acute abscess (April 2005); (C) postsurgical incision and drainage (June 2005). 
physical examination the patient had a $5 \times 5-\mathrm{cm}$ fluctuant noninflamed, nontender nodule on his right cheek. An incision and drainage procedure was performed and approximately $50 \mathrm{~mL}$ of yellow, grainy, purulent contents was drained. Anaerobic culture of the material grew $2+P$. acnes while routine culture grew $1+$ coagulase-negative Staphylococcus. Surgical pathology analysis revealed numerous fragments of foreign material and acute and chronic inflammation consistent with abscess. Gram stain revealed scattered gram positive cocci in singlets, doublets, and chains with streptococcal species being the presumed organism. Mycobacterial and fungal stains were negative. The abscess had completely resolved with antibiotic therapy at 2 weeks of follow-up with return of visible lipoatrophy on the right side (Figure 4).

\section{Case 5}

A 42-year-old man with stable HIV infection received Bio-Alcamid injections over two sessions in June 2005. He presented in October 2006 with
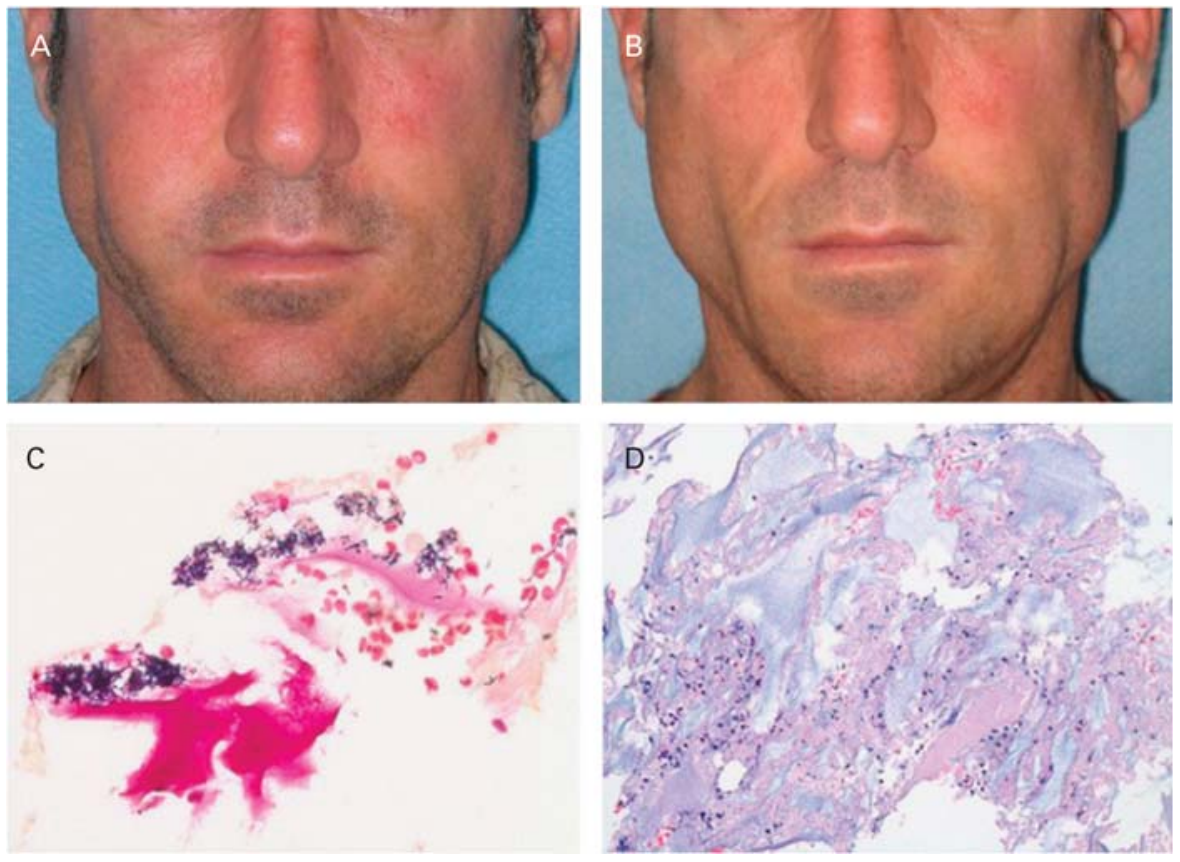

Figure 4. Case 4: (A) presentation of chronic abscess; (B) 1 week postsurgical incision and drainage; (C) Gram stain revealing scattered Gram-positive cocci in singlets, doublets, and chains with streptococcal species being the presumed organism; (D) numerous fragments of foreign material and acute and chronic inflammation consistent with abscess. Original magnification, (C) $\times 400,(D) \times 200$. 
required prolonged IV antibiotic therapy to achieve remission. Local oral streptococcal bacteria may be capable of directly invading the implant material. It is also possible, as in Case 1, that the bacteria may be seeded within the implant by way of needle puncture during a dental or surgical procedure. The inner portions of these balloon-like implants may lack the necessary host defenses to ward off infection. A recent analysis over 3 years of 2,000 cases of Bio-Alcamid treatments for a variety of cutaneous atrophies revealed that 12 of 2,000 implants became infected with $S$. aureus, requiring drainage of the implant and antibiotic therapy in all 12 cases. $^{3}$ Both physicians and patients should be aware of the possible risk of late-appearing abscesses after Bio-Alcamid injections. Furthermore, these cases suggest a rationale for prophylactic antibiotic therapy before dental or surgical procedures in patients who have received BioAlcamid injections.

\section{References}

1. Jones D. HIV facial lipoatrophy: causes and treatment options. J Dermatol Surg 2005;31(Suppl 4):1519-29.

2. Jones D, Carruthers A, Orentreich D, et al. Highly purified 1000cSt silicone oil for treatment of human immunodeficiency virusassociated facial lipoatrophy: an open pilot trial. J Dermatol Surg 2004;30:1279-86.

3. Pacini S, Ruggiero M, Morucci G, et al. Bio-Alcamid: a novelty for reconstructive and cosmetic surgery. Ital J Anat Embryol 2002;107:209-14.

4. Pacini S, Ruggiero M, Morucci G, et al. Bio-Alcamid, a novel prosthetic polymer, does not interfere with morphological and functional characteristics of human skin fibroblasts. Plast Reconstr Surg 2003;111:489-91.

5. Protopapa C, Giuseppe S, Caporale D, Cammarota N. Bio-Alcamid $^{\mathrm{TM}}$ in drug-induced lipodystrophy. J Cosmet Laser Ther 2003;5:226-30.

6. Treacy P, Goldberg D. Use of a biopolymer polyalkylimide filler for facial lipodystrophy in HIV-positive patients undergoing treatment with antiretroviral drugs. J Dermatol Surg 2006; 32:804-8.

Address correspondence and reprint requests to: Derek H. Jones, MD, 9201 Sunset Blvd., \#602, Los Angeles, CA 90069, or e-mail: derekjonesmd@aol.com 